\title{
Comparison of enzyme Immunoassay with Immunofluorescence Assay for the Diagnosis of Mycoplasma pneumoniae Respiratory Tract Infections in Children
}

\author{
Osama Mohammed Saed Abdul-Wahab \\ Department of Microbiology, Faculty of Medicine, King Khalid University, Saudi Arabia
}

Received 2013-06-09, Revised 2013-06-20; Accepted 2013-06-21

\begin{abstract}
Mycoplasma pneumoniae is a frequent cause of acute respiratory infections in both children and adults. Currently, diagnosis of $M$. pneumoniae infection will based principally on serology and the detection of IgM can provide an early and sensitive diagnosis in children. A variety of commercial immunodiagnostic assays, such as Indirect Enzyme-Linked Immunosorbent (ELISA) assay and Indirect Immunofluorescence (IFA), are now available as serological methods. The Aim of this comparative prospective study, was to compare two different approaches to the rapid detection of Mycoplasma pneumoniae respiratory tract infections among children. For this purpose, a commercial IFA assay for detection of M. pneumoniaespecific IgM antibody in acute-phase sera was used to compare with ELISA assay for specific-IgM antibody and to sought wither the use of either serological assays as a more reliable laboratory diagnosis for Mycoplasma pneumoniae respiratory tract infections in children. This study was designed as a comparative prospective study in which 90 patients (Mean age of the patients in case group was $5.94 \pm 2.73$ and in control group was 6.51 \pm 2.26 ) of either sexes were included. These patients were classified into two groups: first group (case group), included 45 patients who had been admitted in hospital with diagnosis of respiratory tract infections and the second group (control group), included 45 healthy patients who had no history of respiratory tract infections. Both the groups were age and sex matched. Presence of IgM antibodies to Mycoplasma pneumoniae was assessed by IFA kit assay in both groups and the detection result was compared with result of previous study using ELISA assay. In the case group, 2 (4\%) cases out of 45 children were positive for antimycoplasma antibody whereas in the control group, all children were negative. All positive case group patients had symptoms of acute pneumonia. $18(40 \%)$ of the patients were diagnosed with bronchial asthma (40\%) inclusive of all the two cases diagnosed with Mycoplasma pneumoniae infection. The IgM-positive rate for IFA assay (4\%) was lower than in ELISA assay (9\%), as compared from both kits. Taking the ELISA assay as a gold standard for the presence of Mycoplasma pneumoniae respiratory tract infections, the sensitivity, specificity and positive and negative predictive values of the IFA assay in comparison to ELISA, were 50 (CI:8.30 to $91.70 \%$ ), 100 (CI:91.31 to $100 \%$ ), 100 and $95.34 \%$ respectively. The results of IgM IFA assay showed relatively lower positivity than ELISA assay in the early acute phase, the results of IFA undertaken for the detection of M. pneumoniae respiratory tract infections and it is a first study in the Saudia Arabia of its kind from the region reporting such a disease in children using a serological assays as IFA and ELISA assays for comparasion. We therefore conclude that the use of ELISA assay conducted in the country as a more reliable laboratory diagnosis for Mycoplasma pneumoniae respiratory tract infections in children. Further future studies need to be carried out to investigate the use of PCR to allow fast and reliable diagnosis of $M$. pneumoniae infection during the early phases of infection in children.
\end{abstract}

Keywords: Immunofluorescent Assay (IFA), Mycoplasma Pneumoniae, Enzyme Immunoassay (ELISA), Respiratory Tract Infections, Children 


\section{INTRODUCTION}

Mycoplasma pneumoniae is a frequent cause of acute respiratory infections in both children and adults. This organism is difficult to culture because of its fastidious nature and growth is too slow for diagnostic use. A number of assays have been used for detection and quantification of M. pneumoniae antibodies (Tully et al., 1996), including the older ones based on Complement Fixation (CF), metabolic inhibition (e.g., tetrazolium reduction inhibition technique), mycoplasmacidal test, radioimmunoprecipitation, radioimmunoassay and hemagglutination (Taylor-Robinson et al., 1966; Busolo and Meloni, 1983; Hu et al., 1983) and the newer techniques based on enzyme immunoassay (Jacobs, 1993), Those relying on culture are too cumbersome and slow for modern diagnostic services, however, CF (which predominantly detects IgM) and EIA are widely used and particle agglutination assays have become available (Tully et al., 1996). Currently, diagnosis of $M$. pneumoniae infection will based principally on serology and the detection of IgM can provide an early and sensitive diagnosis in children. A variety of commercial immunodiagnostic assays, such as ELISA and immunofluorescence, are now available as diagnostic serological methods.

Apart from our recent study, which will revealed the detection of M. pneumoniae specific IgM antibodies to the children patients which is a first study of its kind from the region reporting such a disease in children using a serological assay as ELISA and validated the utility of this methodology in terms of the acute phase diagnosis using single point serum sample for $M$. pneumoniae respiratory tract infections specifically in children (Abdul-Wahab et al., 2013), additionally, in other study, detection of M. pneumoniae specific IgG antibodies to $M$. pneumoniae in adults patients with ischemic heart disease was also assessed by ELISA method and confirmed the reported study in Saudia Arabia (Abdul-Wahab et al., 2012), However, other studies that have been published conducting serological assays for the diagnosis of $M$. pneumoniae, are very little that conducted in this country concerning the association of Mycoplasma pneumoniae with respiratory tract infections in children and in one a study published by (Al Rashed, 1998), it was found that Mycoplasma pneumoniae IgM and IgG antibodies each of the sera were collected and tested by IFAT, using commercial kits, the organism to be the second most common causative agent after Respiratory Syncytial Virus
(RSV) accounting for $9 \%$ of all cases of 511 children with acute respiratory tract infection.

The Aim of this comparative prospective study, was to compare two different approaches to the rapid detection of Mycoplasma pneumoniae respiratory tract infections among children. For this purpose, a commercial IFA assay for detection of M. pneumoniaespecific IgM antibody in acute-phase sera was used to compare with ELISA assay for specific-IgM antibody from the same patients sera of recently reported association of Mycoplasma pneumoniae respiratory tract infections (Abdul-Wahab et al., 2013) and to sought wither the use of either serological assays as a more reliable laboratory for diagnosis of Mycoplasma pneumoniae respiratory tract infections in children.

\section{MATERIALS AND METHODS}

This study was designed as a case-control study, in which 90 patients (Mean age of the patients in case group was $5.94 \pm 2.73$ and in control group was $6.51 \pm 2.26)$ of either sexes were included.The study conducted during one year (January, 2012-January, 2013) in Peadiatric ward at Asser Central Hospital in Abha (a city in southwest region of Saudi arabia). These patients were classified into two groups: first group (case group), included 45 patients who had been admitted in hospital with diagnosis of respiratory tract infections and the second group (control group), included 45 healthy patients who had no history of respiratory tract infections. Both the groups were age and sex matched. Presence of IgM antibodies to Mycoplasma pneumoniae was assessed by Indirect Immunofluorescent (IFA) kit assay in both groups and the detection result was compared with results of the previous study using the M. pneumoniae ELISA assay (Abdul-Wahab et al., 2013) and to sought wither the use of either serological assays as a more reliable laboratory diagnosis of Mycoplasma pneumoniae. IgM against M.pneumoniae were assessed using the IFA assay technique for IgM (Microbiologists, Vircell) and detecting IgM specific antibodies against M pneumonia was performed on a first serum sample in accordance to manufacturer's guidelines (Microbiologists, Vircell). Slides were interpreted according to the manufacturer's instructions. All our information was Statistically analyzed by using the Statistical Package for the Social Sciences version 13.0 (SPSS software, Inc., Chicago, IL, USA). SPSS software and for statistic analysis, t-test and chi square test was used. Results with $\mathrm{P}$ value less than 0.05 was considered as statistically significant. 


\section{RESULTS}

Ninety patients were studied. Mean age of the patients in case group was $5.94 \pm 2.73$ and in control group was $6.51 \pm 2.26)$. There was not significant statistic difference for age between two groups $(\mathrm{p}=0.08)$ and age groups between male and females patients were not statistically different. In the case group, patients with diagnosis of respiratory tract infections such as pneumoniae and bronchial asthma, 2 (4\%) cases out of 45 children were positive for anti-mycoplasma antibody whereas in the control group, all children were negative. All positive case group patients had symptoms of acute pneumonia. $18(40 \%)$ of the patients were diagnosed with bronchial asthma (40\%) inclusive of all the two cases diagnosed with Mycoplasma pneumoniae infection (Table 1), (Fig. 1).

Results of $M$. pneumoniae detection from the two assays were compared (Table 2). IgM was positive in two acute-phase serum specimens of the total of 45 childrens were tested with IFA assay for the presence of $M$. pneumoniae infection, the positive rate $(4 \%)$ was lower than in ELISA assay (9\%), as compared from both kits.
Table 1. Antimycoplasma antibody in case and control groups $(\mathrm{n}=45 ; \mathrm{n}=45)$

\begin{tabular}{llll}
\hline & Antimycoplasma $\mathrm{Ab}$ & \\
& ---1 & \\
Group: & Positive & Negative & Total \\
\hline Case: & 2 & 43 & 45 \\
Control: & 0 & 45 & 45 \\
\hline$P=0.1522$ & & &
\end{tabular}

$\mathrm{P}=0.1522$

Table 2. Result of M. pneumoniae detection and serology in 45 children group with respiratory tract infections

\begin{tabular}{lcc}
\hline & Acut-phase serum specimen & \\
Patient & -------------------------- \\
$\mathrm{n}=45$ & IgM-IFA* & IgM-ELISA** \\
\hline Positive: & 2 & 4 \\
Negative: & 43 & 41 \\
Total: & 45 & 45 \\
\hline
\end{tabular}

IFA: Immunofluorescence assay, ELISA: Enzyme-linked immunosorbent assay *Positive reaction=abundant apple-green fluorescent aggregates of mycoplasma on background of red stained cells can be observed, Negative reaction $=$ red cellular pattern can be observed **: Antibody index $=$ (sample O.D/cutoff serum mean O.D.) $\times 10$, Index $:<9=$ Negative, $9-11$ $=$ Equivocal, $>11=$ Positive

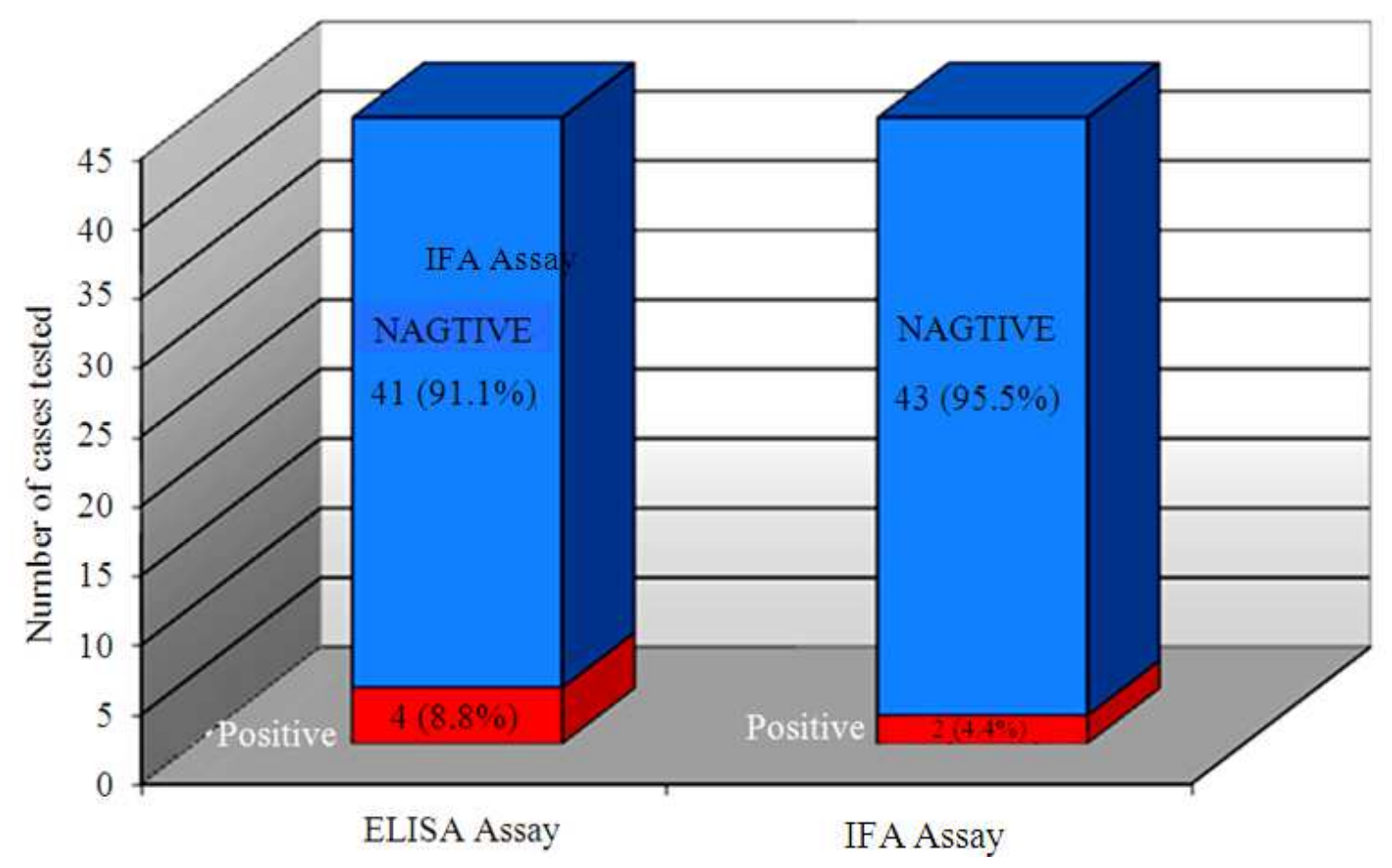

Fig. 1. ELISA assay in comparison to IFA assay for diagnosing M. pneumoniae in Children with respiratory tract infections 


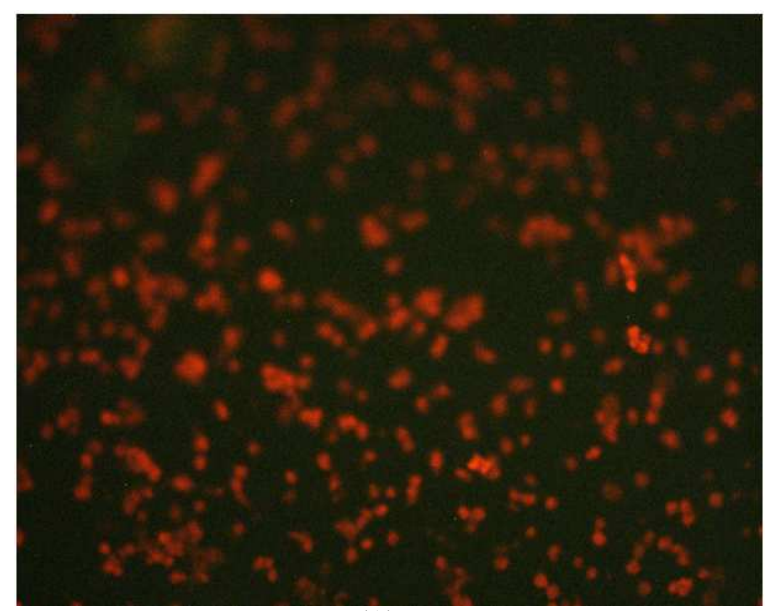

(A)

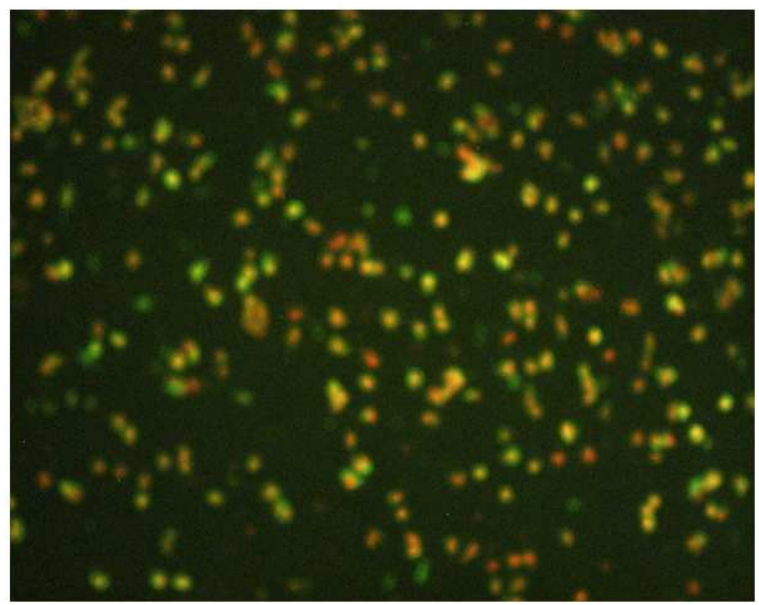

(B)

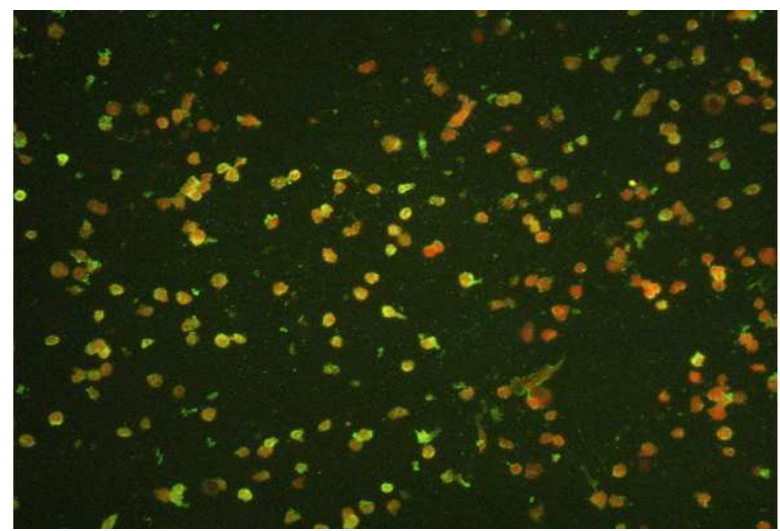

(C)

Fig. 2. Immunofluorescence Assay (IFA) for detection M. pneumoniae IgM specific antibody in case group patients, (A) Mycoplasma negative Control: Well of slide coated with M. pneumoniae, FH strain (ATCC 15531) and treated with normal serum and human anti-Mycoplasma pneumoniae IgM and anti-human IgM fluorescein conjugate.. Red cellular pattern can be observed under a fluorescence microscope. Magnification $=40 \times,(B)$ Mycoplasma positive Control: Well of slide coated with M. pneumoniae, FH strain (ATCC 15531) and treated with human anti-Mycoplasma pneumoniae IgM and anti-human IgM fluorescein conjugate. Abundant apple-green fluorescent aggregates of Mycoplasma pneumoniae IgM specific antibody on background of red stained cells can be observed under a fluorescence microscope. Magnification $=40 \times$, (C) Detected Mycoplasma pneumoniae infection in the case group: Well of slide coated with M. pneumoniae, FH strain (ATCC 15531) and treated with tested serum sample with human anti-Mycoplasma pneumoniae IgM and anti-human IgM fluorescein conjugate. Abundant apple-green fluorescent aggregates of Mycoplasma pneumoniae IgM specific antibody on background of red stained cells can be observed under a fluorescence microscope. Magnification $=40 \times$

Taking the ELISA assay as a gold standard for the presence of Mycoplasma pneumoniae respiratory tract infections, the sensitivity, specificity and positive and negative predictive values of the IFA assay in comparison to ELISA assay, were 50 (CI:8.30\% to 91.70\%), 100 (CI:91.31\% to $100 \%$ ), 100 and $95.34 \%$ respectively. Figure 2 showed the IFA assay for detection $M$. pneumoniae $\operatorname{IgM}$ specific antibody in case group patients (Fig. 2).

\section{DISCUSSION}

Mycoplasma pneumoniae is a common cause of community-acquired pneumonia. Pneumonia may be the most severe manifestation of respiratory $M$. pneumoniae infection. The most typical symptoms in children are cough and wheezing, which are often accompanied by upper respiratory tract manifestations mimicking viral respiratory syndromes (Sorensen et al., 2013). The 
pathogen can produces diseases of varied severity ranging from mild upper respiratory tract infection to sever atypical pneumoniae, although it is rarely fatal, the pathogen is an important cause of acute respiratory tract infection especially as a potential etiology of the clinical entity termed "atypical pneumoniae" this organism is also responsible for producing a wide spectrum of nonpulmonary manifestations including neurological, hepatic, cardiac diseases, hemolytic anemia, polyartritis and eryythema multiforme (Kashyap and Sarkar, 2010).

Many diagnostic tests for $M$. pneumoniae infection have been proposed. The standard laboratory methods for the diagnosis of $M$. pneumoniae infections have been culture and serology (Waris et al., 1998), However, growing of $M$. pneumoniae in culture is difficult and time consuming (Atkinson et al., 2008). The IFA assay, have been developed to detect antibodies to $M$. pneumoniae. The test provide accurate, quantitative serologic results but interpretation of the data generated is subjective and requires fluorescence microscopy (Waiters and Talkington, 2004), However, the test has several advantageous features include its reproducibility which can be maintained, antibiotics in serum do not produce spurious results in the same way that they might in a metabolism-inhibition test where the inhibitory effect of an antibiotic might be mistaken for that of antibody, the test is quick to perform , and relatively easy to read (Taylor-Robinson, 1996). Enzyme Immunoassay (ELISA) on the other hand, is currently the most widelyused serological method. After its discovery, several commercial kits have been produced with a great variety of antigen preparations, such as glycolipids, purified proteins (including $\mathrm{P} 1$ adhesin), synthetic peptides and a mix of antigen. It was appeared that the test is more sensitive for the detection of acute infection than cultures and has sensitivity comparable to PCR and is highly specific (Cassel et al., 1996; Vervloet et al., 2007; Atkinson et al., 2008), It allow the detection of IgG and/or IgM antibodies and apparently too sensitive for detecting specific antibodies. The technique is automated and requires small amounts of serum (Andreu et al., 2006; Vervloet et al., 2007). Furthermore, EIAs can detect IgG and $\operatorname{IgM}$ separately to distinguish between current and past infections (Talkington et al., 2004; Beersma et al., 2005).

In the present study, our results showed detection of M. pneumoniae-specific IgM in two positive acute-phase serum specimens out of the total of 45 children were tested with IFA assay for the presence of $M$. pneumoniae infection, the positive rate $(4 \%)$ was lower than in ELISA assay (9\%), as compared from both kits, Although this number of $M$. pneumoniae-positive patients was relatively small with IFA assay in comparison to ELISA assay, methods for the early diagnosis of $M$. pneumoniae are limited because of the lack of $\operatorname{IgM}$ antibodies using a variety of serologic assays (Atkinson et al., 2008; You-Sook and Kyung-Yil, 2012), However, this study showed results from IgM ELISA demonstrated relatively high positivity in the early acute phase of $M$. pneumoniae infection which in agreement with several other studies (Cassel et al., 1996; Myeong et al., 2012; Abdul-Wahab et al., 2013).

In our study, taking the ELISA assay as a gold standard for the presence of Mycoplasma pneumoniae respiratory tract infections, the sensitivity, specificity and positive and negative predictive values of the IFA assay in comparison to ELISA, were 50 (CI:8.30\% to $91.70 \%$ ), 100 (CI:91.31\% to $100 \%$ ), 100 and $95.34 \%$ respectively. Sensitivities of serologic assays for the detection of Mycoplasma pneumoniae antibodies are known to vary (Beersma et al., 2005; Loens et al., 2010), IgM ELISA used in our previous study was found to be the most sensitive and specific (Abdul-Wahab et al., 2013), which is in agreement with the results obtained by other studies (Waris et al., 1998; Dorigo-Zetsma et al., 1999; Souliou et al., 2007; Vervloet et al., 2007; Myeong et al., 2012), IFA assay on the other hand, was found more sensitive and specific in adults (Andreu et al., 2006), the assay is relatively easy to perform and gives quantitative results but the main limitations are subjectivity of the interpretation of results and cross-reaction with rheumatoid factor (Vervloet et al., 2007).

In the present study, although the ELISA assay was found to be most sensitive and specific than IFA assay in children, However, a significant proportion of patients was not diagnosed at the initial visit using either assay alone. The ELISA specificity was found to be high, but it was found that the specificity of commercial kits, in general, is not always sufficient (Souliou et al., 2007) and it has been suggested that these kits need further improvement and that serology in a single serum sample is not always enough (Nir-Paz et al., 2006; Souliou et al., 2007). It has been concluded in reported study that combination of Polymerase Chain Reaction (PCR) and IgM ELISA is highly sensitive for rapidly diagnosing $\mathrm{M}$. pneumonia infection in children during the acute phase (Waris et al., 1998; Souliou et al., 2007). This combination has been revealed in a study with the positive results for all 12 cases with definite infection during the early phase of disease (in five cases of both 
methods were positive, in four cases only PCR was positive and in three only $\mu$-capture ELISA was positive). Therefore, the sensitivity will increased to $100 \%$ (Souliou et al., 2007). The slow growth of $M$. pneumoniae coupled with lack of suitable commercially available culture media will limits the usefulness of cultural diagnosis and results in reliance on serology and the one of the newer techniques like PCR (Cassel et al., 1996), In the latter method, a nasal or throat swab specimens were analysed for the presence of $M$. pneumoniae and found it is an easy, reliable and quick diagnostic test in infants and children (Nilsson et al., 2008; Sorensen et al., 2013). We therefore conclude that IgM IFA assay showed relatively lower positivity than ELISA assay in the early acute phase and the use of the commercial ELISA assay in the country as a more reliable laboratory for the accurate and cost-efficient tool for diagnosis of specific IgM antibodies for Mycoplasma pneumoniae respiratory tract infections in children. We believe that further future studies need to be carried out to investigate the PCR as superior to serology to allow fast and reliable diagnosis of $M$. pneumoniae infection during the early phases of infection in children.

\section{CONCLUSION}

The results of our study for the comparison of ELISA and IFA assys demonstrate that detection of $M$. pneumoniae respiratory tract infections and it is a first study in the Saudia Arabia of its kind from the region for reporting such a disease in children using a serological assays for comparasion. The IgM IFA assay showed relatively lower positivity than ELISA assay in the early acute phase, We therefore conclude that use of ELISA assay as a good alternative to the IFA assay and as a more reliable laboratory diagnosis for Mycoplasma pneumoniae respiratory tract infections in children. Further future studies are need to be carried out in order to investigate the PCR as superior to serology to allow fast and reliable diagnosis of $M$. pneumoniae infection during the early phases of infection.

\section{ACKNOWLEDGMENT}

The researcher wish to thank Mr. Majdi A.Mahfuz and Mrs. Beena Grace Charly, from the Serology Section, Asser Central hospital for their help support.We also thank Dr. Ashish Kumar from Faculty of Medicine for his support regarding Statistic Analysis.

\section{REFERENCES}

Abdul-Wahab, O.M.S., A.H. Alsheri, A.S. Assiri, A. Almasswary and F. Benahmed, 2012. Association of Mycoplasma pneumoniae infection with ischemic heart diseases. Am. J. Immun., 8: 117-122. 10.3844/ajisp.2012.117.122

Abdul-Wahab, O.M.S., A.M. Al-Hakami, A.A. shati, A.M. Alsuheel and A. Kumar et al., 2013. Association of mycoplasma pneumoniae with respiratory tract infections in children. Am. J. Immun., 9: 1-8. DOI: 10.3844/ajisp.2013.1.8

Al Rashed, A., 1998. Role of Mycoplasma pneumoniae in acute respiratory-tract infections in Saudi paediatric patients. Ann. Trop. Med. Parasitol., 92: 595-601. PMID: 9797833

Andreu, L.M., A.S. Molinos, R.G. Fernandez, S.V. Gonzalez and R.V. Ausina 2006. Serologic diagnosis of Mycoplasma pneumoniae infections. Enferm Infect. Microbiol. Clin., 24: 19-23. PMID: 17125664

Atkinson, T.P., M.F. Balish and K.B. Waites, 2008. Epidemiology, clinical manifestations, pathogenesis and laboratory detection of Mycoplasma pneumoniae infections. FEMS Microbiol. Rev., 32: 956-973. DOI: 10.1111/j.1574-6976.2008.00129.x

Beersma, M.F., K. Dirven and A.P.V. Dam, 2005. Evaluation of 12 commercial tests and the complement fixation test for Mycoplasma pneumoniae-specific Immunoglobulin $\mathrm{G}(\mathrm{IgG})$ and IgM antibodies, with PCR used as the "gold standard. J. Clin. Microbiol., 43: 2277-2285. DOI: 10.1128/JCM.43.5.2277-2285.2005

Busolo, F. and G.A. Meloni, 1983. Serodiagnosis of $M$. pneumoniae infections by Enzyme-Linked Immunosorbent Assay (ELISA). Yale J. Biol. Med., 56: 517-521. PMID: 6433571

Cassel, G.H., G. Gambill and L. Duffy, 1996. ELISA in Respiratory Infections of Humans. In: Molecular and Diagnostic Procedures in Mycoplasmology: Diagnostic Procedures, Tully, J.G. and S. Razin, (Eds.), Academic Press, Inc. California, USA., ISBN-10: 0080536794, pp: 123-136.

Dorigo-Zetsma, J.W., S.A.J. Zaat, P.M.E. Wertheimvan Dillen, L. Spanjaard and J. Rijntjes et al., 1999. Comparison of PCR, culture and serological tests for diagnosis of mycoplasma pneumoniae respiratory tract infection in children. J. CLin. Microbiol., 37: 14-17. 
Hu, P.C., D.A. Powell, F. Albright, D.E. Gardner and A.M. Collier, 1983. A solid-phase radioimmunoassay for detection of antibodies against Mycoplasma pneumonia. J. Clin. Lab. Immunol., 11: 209-213. PMID: 6422044

Jacobs, E., 1993. Serological diagnosis of Mycoplasma pneumoniae infections: A critical review of current procedures. Clin. Infect. Dis., 17: S79-S82. PMID: 8399943

Kashyap, S. and M. Sarkar, 2010. Mycoplasma pneumonia: Clinical features and management. Lung Ind., 27: 7585. DOI: $10.4103 / 0970-2113.63611$

Loens, K., H. Goossens and M. Ieven, 2010. Acute respiratory infection due to Mycoplasma pneumoniae: Current status of diagnostic methods. Eur. J. Clin. Microbiol. Infect. Dis., 29: 1055-1069. DOI: $10.1007 / \mathrm{s} 10096-010-0975-2$

Myeong, H.K., S.Y. Kang and I.L. Woo, 2012. Comparison of two enzyme immunoassays for detecting mycoplasma pneumoniae. Labmedicine, 43: 74-77.

Nilsson, A.C., P.R. Bjorkman and K.H. Persson, 2008. Polymerase chain reaction is superior to serology for the diagnosis of acute Mycoplasma pneumoniae infection and reveals a high rate of persistent infection. BMC Microbiol., 8: 93-93. DOI: 10.1186/1471-2180-8-93

Nir-Paz, R., A. Michael-Gayego, M. Ron and C. Block, 2006. Evaluation of eight commercial tests for Mycoplasma pneumoniae antibodies in the absence of acute infection. Clin. Microbiol. Infect., 12: 685688. DOI: 10.1111/j.1469-0691.2006.01469.x

Sorensen, C.M., K. Schønning and V. Rosenfeldt, 2013. Clinical characteristics of children with Mycoplasma pneumoniae infection hospitalized during the Danish 2010-2012 epidemic. Dan. Med. J., 60: A4632. PMID: 23673264

Souliou, E., M. Almasri, A. Papa, A. Theodoridou and E. Diza, 2007. Laboratory diagnosis of Mycoplasma pneumoniae respiratory tract infections in children. Eur. J. Clin. Microbiol. Infect. Dis., 26: 513-515. DOI: $10.1007 / \mathrm{s} 10096-007-0326-0$
Talkington, D.F., S. Shott and M.T. Fallon, 2004. Analysis of eight commercial enzyme immunoassay tests for detection of antibodies to Mycoplasma pneumoniae in human serum. Clin. Diagn. Lab. Immunol., 11: 862-867. DOI: 10.1128/CDLI.11.5.862-867.2004

Taylor-Robinson, D., 1996. Microimmunofluorescence. In: Molecular and Diagnostic Procedures in Mycoplasmology: Diagnostic Procedures, Tully, J.G. and S. Razin (Eds.), Academic Press, San Diego, London, ISBN-10: 0080536794, pp: 147-150.

Taylor-Robinson, D., O. Sobeslavsky, K.E. Jensen, L.B. Senterfit and R.M. Chanock, 1966. Serologic response to Mycoplasma pneumoniae infection1 Evaluation of immunofluorescence, complement fixation, Indirect haemagglutination and tetrazolium reduction inhibition tests for the diagnosis of infection. Am. J. Epidemiol., 83: 287-298.

Tully, J.G. and S. Razin, 1996. Molecular and Diagnostic Procedures in Mycoplasmology: Diagnostic Procedures. 2nd Edn., Academic Press, Inc. California, USA., ISBN-10: 0125838069, pp: 466.

Vervloet, L.A., C. Marguet and P.A.M. Camargos, 2007. Infection by mycoplasma pneumoniae and its importance as an etiological agent in childhood community-acquired pneumonias. Brazilian J. Infect. Dis., 11: 507-514. PMID: 17962878

Waiters, K.B. and D.F. Talkington, 2004. Mycoplasma pneumoniae and its role as a human pathogen. Clin. Microbiol. Rev., 17: 697-728. DOI: 10.1128/CMR.17.4.697-728.200

Waris, M.E., P. Toikka, T. Saarinen, S. Nikkari and O. Meurman et al., 1998. Diagnosis of Mycoplasma pneumoniae pneumonia in children. J. Clin. Microbiol., 36: 3155-3159. PMID: 9774556

You-Sook, Y. and L. Kyung-Yil, 2012. Mycoplasma pneumoniae pneumonia in children. Korean J. Pediatr, 55: 42-47. DOI: 10.3345/kjp.2012.55.2.42 